\title{
Effects of dihydrotestosterone on skeletal muscle transcriptome in mice measured by serial analysis of gene expression
}

\author{
M Yoshioka, A Boivin, P Ye, F Labrie and J St-Amand \\ Molecular Endocrinology and Oncology Research Center, Laval University Medical Center and Department of Anatomy and Physiology, Laval University, \\ 2705 Boulevard Laurier, Ste-Foy, Québec G1V 4G2 Canada
}

(Requests for offprints should be addressed to J St-Amand; Email: Jonny.St-Amand@ crchul.ulaval.ca)

\begin{abstract}
In order to characterize the action of androgen in skeletal muscle, we have investigated the effects of castration (GDX) and dihydrotestosterone (DHT) on global gene expression in mice. The serial analysis of gene expression method was performed in the muscle of male mice in six experimental groups: intact, GDX and GDX+DHT injection 1, 3, 6 or $24 \mathrm{~h}$ before they were killed. A total of 780822 sequenced tags quantified the expression level of 80142 tag species. Thirteen and seventy-nine transcripts were differentially expressed in GDX and DHT respectively $(P<0.05)$, including eight partially characterized and 21 potential novel transcripts. The induced transcripts within $3 \mathrm{~h}$ after DHT injection were involved in the following functions: transcription, protein synthesis, modification and degradation, muscle contraction and relaxation, cell signaling, polyamine biosynthesis, cell cycle progression and arrest, angiogenesis, energy metabolism and immunity. However, the inductions of transcripts related to cell cycle arrest and angiogenesis were no longer significant $24 \mathrm{~h}$ after DHT injection. The current study might suggest that DHT promotes protein synthesis, cell signaling, cell proliferation and ATP production, as well as muscle contraction and relaxation at the transcriptional level in skeletal muscle in vivo.
\end{abstract}

Journal of Molecular Endocrinology (2006) 36, 247-259

\section{Introduction}

Sarcopenia, characterized by a loss of muscle mass, strength and endurance, occurs with aging and chronic medical disorders such as human immunodeficiency virus (HIV) infection and long-term systemic glucocorticoid (Gcc) therapy. On the other hand, testosterone supplementations in older men and HIV-infected men with weight loss who have low testosterone concentrations (Bhasin et al. 2001) as well as men requiring long-term systemic Gcc treatment (Truhan \& Ahmed 1989) increase fat-free mass and muscle strength.

Skeletal muscle is one of the target tissues for the anabolic action of androgens. Androgen receptors (AR), localized in the muscle cells as well as fat, nerve and mesenchymal pluripotent cells which reside in the muscle tissue, likely mediate the effects of androgens by increasing muscle mass, protein synthesis, ribosomal contents, mitochondrial areas, myonuclear number, satellite cell number and myogenesis of pluripotent mesenchymal cells while decreasing protein degradation and adipogenesis of the pluripotent mesenchymal cells (Herbst \& Bhasin 2004). Upon the ligand binding to intracellular $\mathrm{AR}$, the androgen- $\mathrm{AR}$ complex is translocated to the nucleus and binds to specific DNA sequences, androgen response elements, which results in transcription of specific genes (Michel \& Baulieu 1980, Simental et al. 1991). Androgens also have rapid non-genomic actions in the muscle (Estrada et al. 2000, 2003), including membrane receptor coupled to $G$ protein, inositol 1,4,5-trisphosphate $\left(\mathrm{IP}_{3}\right)$ receptor, calcium ion $\left(\mathrm{Ca}^{2+}\right)$ and mitogen-activated protein kinase (MAPK)/extracellular signal-regulated kinase (ERK) phosphorylation cascade. In addition to its role in muscle contraction, intracellular $\mathrm{Ca}^{2+}$ is thought to regulate gene expression in the skeletal muscle (Estrada et al. 2001, Araya et al. 2003). Thus, both genomic and non-genomic actions of androgens are responsible for the transcription of androgen responsive genes (ARG).

Circulating testosterone is transformed into its bioactive metabolite, dihydrotestosterone (DHT) by $5 \alpha$-reductase in target tissues. DHT is one of the most potent natural androgens. However, the molecular mechanisms of the anabolic effect of androgens in skeletal muscle are poorly understood. With the advent of serial analysis of gene expression (SAGE) (Velculescu et al. 1995), new possibilities have arisen for large scale transcriptome analysis. Using this method, we have previously studied the molecular mechanisms responsible for muscle atrophy caused by immobilization in rats (St-Amand et al. 2001), as well as the gene expression profile of endurance-trained men (Yoshioka et al. 2003). 
In the present study, we have investigated the effects of castration (GDX) and DHT on global gene expression in the skeletal muscle of male mice using the SAGE strategy. DHT-modulated transcripts are involved in $\mathrm{Ca}^{2+}$ release, cell signaling, cell proliferation, mRNA and protein synthesis and energy metabolism. These findings constitute a first step towards a precise understanding of the molecular mechanisms involved in the physiological effects of androgens in skeletal muscle.

\section{Materials and methods}

\section{Sample preparation}

The right gastrocnemius muscle was obtained from C57BL6 mice of 12 to 14 weeks of age purchased from Charles River Canada Inc. (St-Constant, QC, Canada). The animals were housed with lights on from 0715 to 1915 h, and had access to Lab Rodent Diet No. 5002 (Ren's Feed and Suppliers, Oakville, ON, Canada) and water ad libitum. No treatment was performed on 26 intact mice. GDX was performed 7 days prior to organ collection in each of the 14 mice from the GDX and DHT groups. Mice of the GDX group received an i.p. injection of vehicle solution $(0 \cdot 4 \%(\mathrm{w} / \mathrm{v})$ Methocel A15 LV Premium/5\% ethanol; Dow Chemicals Co, Laval, Quebec, Canada) $24 \mathrm{~h}$ before they were killed, while a physiological dose of DHT $(0.1 \mathrm{mg} /$ mouse $)$ was injected 1, 3, 6 and $24 \mathrm{~h}$ prior to their being killed (DHT $1 \mathrm{~h}$, DHT $3 \mathrm{~h}$, DHT $6 \mathrm{~h}$ and DHT $24 \mathrm{~h}$ groups). All mice were killed between 0830 and $1230 \mathrm{~h}$ by cervical dislocation and decapitation under isoflurane anesthesia. The right gastrocnemius muscle was sampled from each mouse and pooled together for analysis of the same group to eliminate inter-individual variations and to extract sufficient amounts of mRNA. The tissues were stored at $-80{ }^{\circ} \mathrm{C}$ until RNA extraction. All animal experimentation was conducted in accordance with the requirements of the Canadian Council on Animal Care.

\section{Transcriptome analysis}

The SAGE method was performed as previously described (Velculescu et al. 1995, St-Amand et al. 2001). Total RNA was isolated by Trizol (Invitrogen Canada Inc., Burlington, ON, Canada). The quality of total RNA was monitored by micro-capillary electrophoresis (Bioannalyzer 2100; Agilent Technologies, Mississauga, ON, Canada). Polyadenylated RNA was extracted with the Oligotex mRNA Mini Kit (Qiagen Inc., Mississauga, ON, Canada), annealed with the biotin- $5^{\prime}-\mathrm{T}_{18}-3^{\prime}$ primer and converted to cDNA using the cDNA synthesis kit (Invitrogen Canada Inc.). The resulting cDNAs were digested with NlaIII (New England BioLabs Ltd, Pickering, ON, Canada) and the $3^{\prime}$ restriction fragments were isolated with streptavidin-coated magnetic beads
(Dynal Biotech LLC, Brown Deer, WI, USA) and separated into two populations. Each population was ligated to one of two annealed linkers and extensively washed to remove unligated linkers. The tag beside the most $3^{\prime}$ 'NlaIII restriction site (GATG) of each transcript was released by digestion with BsmFI (New England BioLabs Ltd). The blunting kit from Takara Bio Inc. (Otsu, Japan) was used for the blunting and ligation of the two tag populations. The resulting ligation products containing the ditags were amplified by PCR and digested with NlaIII. The band containing the ditags was extracted from the $12 \%$ polyacrylamide gel with Spin-X microcentrifuge tube (Fisher, Pittsbergh, PA, USA). The purified ditags were self-ligated to form concatemers using T4 ligase (Invitrogen Canada Inc.). The concatemers ranging from $500 \mathrm{bp}$ to $1800 \mathrm{bp}$ were isolated by agarose gel and extracted with Gene-Clean Spin (Qbiogene, Montreal, QC, Canada). The resulting DNA fragments were ligated into the SphI site of pUC19 and cloned into UltraMAX DH5 $\alpha$ FT competent cell (Invitrogen Canada Inc.). White colonies were picked up with a Q-Bot colony picker (Genetix Ltd, New Milton, Hants, UK). Concatemer inserts were sequenced by the Applied Biosystems 3730 (Foster City, CA, USA).

\section{Bioinformatic analysis}

Sequence files were analyzed using the SAGEana program, a modification of SAGEparser (Dinel et al. 2005). In brief, tags corresponding to linker sequences were discarded and replicate concatemers were counted only once. Identification of the transcripts was obtained by matching the $15 \mathrm{bp}$ (sequence at the last CATG + 11 bp tags) with SAGEmap, UniGene and GenBank databases on 5 March 2004. A minimum of one expressed sequence tag (EST) with a known polyA tail had to be in the UniGene cluster to identify the last NlaIII site on the corresponding cDNA. We have previously shown that the SAGE method is very reproducible with $r^{2}=0.96$ between two libraries constructed from the same total RNA pool (Dinel et al. 2005). Classification of the transcripts was based upon the updated information of the genome directory (Adams et al. 1995) found at the TIGR web site (http://www.tigr.org/) as well as previously published papers.

\section{Statistical analysis}

We used the comparative count display test to identify the transcripts significantly differentially expressed $(P \leq 0.05)$ between the groups with more than a twofold change (Lash et al. 2000). The data were normalized to 100000 tags for presentation. 


\section{Results}

The sequencing of 780822 SAGE tags quantified the expression level of $80142 \mathrm{tag}$ species. In total, 13 and 79 transcripts were differentially expressed between intact vs GDX and DHT vs GDX respectively $(P<0 \cdot 05)$. Five transcripts were modulated by both GDX and DHT, in which the effects of GDX on four of these transcripts were counteracted by DHT. GDX removes all testicular hormones, precursors and secreted factors as well as their interactions, whereas we administered only the most potent natural androgen, DHT, in GDX mice. This might therefore explain the discrepancy of the effects of GDX or DHT in the transcripts regulated by only one of these experimental conditions. Moreover, the SAGE method may not have detected the down-regulation of some low expressed transcripts because of a weak statistical power (Dinel et al. 2005). Indeed, $72 \%$ of the DHT up-regulated transcripts were not statistically detected as being regulated by GDX, since the intact group had less than nine tags. On the other hand, the changes in the expression level of some transcripts may be counteracted by a change in other isoforms and transcript species such as we have observed for S-adenosylmethionine decarboxylase 1 (Amdl). This could point to a different mechanism with respect to both up-regulation and suppression.

The transcripts related to muscle contraction, extracellular matrix (ECM), transcription and protein metabolism which were regulated by GDX and DHT are presented in Table 1. The transcripts related to both muscle contraction (triadin $(\operatorname{Trdn})$ ) and relaxation (parvalbumin (Pvalb)) were up-regulated by DHT injection. The expressions of serine (or cysteine) proteinase inhibitor clade A members $1 \mathrm{a}, 1 \mathrm{~b}, 1 \mathrm{e}$ and $3 \mathrm{k}$ (Serpinala, 1b, le and 3k) which inhibit the degradation of ECM components, were decreased by GDX whereas DHT increased EST procollagen type I $\alpha 2$ (EST Colla2). DHT induced the expression of transcription modulators (fragile $\mathrm{X}$ mental retardation gene 2 (Fxr2h), O-linked N-acetylglucosamine transferase (Ogt) and SET and MYND domain containing 2 (Smyd2)). DHT up-regulated the transcripts related to ribosomal proteins (L51 (Mrpl51), L34 (Rpl34) and S20 (Rps20)), chaperones (chaperone ABCl activity of bcl complexlike (Cabcl) and chaperonin subunit 8 (Cct8)) and protein degradation (proteasome $26 \mathrm{~S}$ subunit ATPase 3 (Psmc3) and protease 26S subunit ATPase 5 (Psmc5)), while ribosomal proteins S24 (Rps24) and S27 (Rps27) were down-regulated.

Table 2 shows the differentially expressed transcripts which are involved in cell proliferation and signaling as well as other functions. GDX down-regulated growth arrest and DNA damage-inducible $45 \gamma$ (Gadd45g) and the effect was reversed by DHT treatment. DHT also increased the transcripts related to the cell proliferation, such as aldo-keto reductase family 1 member A4 (Akrla4), Golgi reassembly stacking protein 2 (Gorasp2), pituitary tumor-transforming 1 (Pttgl) and t-complex testis-expressed 1 (Tctex 1 ), while decreasing lectin galactose-binding soluble 1 (Lgals1) which inhibits cell proliferation. Moreover, GDX and DHT modulated key transcripts related to polyamine biosynthesis (ornithine decarboxylase antizyme (Oaz), Amdl and sperminebinding protein $(\mathrm{Sbp}))$. The transcripts related to the cell signaling, such as ankyrin repeat and SOGs boxcontaining protein 5 (Asb5), calcium/calmodulindependent protein kinase II $\gamma($ Camk2g), dual specificity phosphatase 1 (Dusp1), histidine triad nucleotide binding protein 1 (Hintl) and membrane protein palmitoylated 6 (Mpp6) were also induced by DHT. In addition, GDX down-regulated transthyretin (Ttr) whereas DHT up-regulated $\beta 2$-microglobulin $(\mathrm{B} 2 \mathrm{~m})$, CD59a antigen (Gd59a) and dendritic cell protein GA17 (Ga17).

The modulated transcripts related to energy metabolism are presented in Table 3. In the lipid metabolism, the expression of apolipoprotein A-II (Apoa2) was decreased by GDX whereas lipin 1 (Lpin 1) was increased by DHT. GDX also suppressed cytochrome c oxidase 1 (MtCol) expression whereas DHT induced the expression of 18 transcripts related to mitochondrial oxidative phosphorylation (OxPhos) and ATP production with two exceptions (NADH dehydrogenase $3(\mathrm{MtNd} 3)$ and NADH dehydrogenase $1 \beta$ subcomplex 9 (Ndufb9)) which were suppressed by DHT. GDX and DHT also modulated the expression of seven partially characterized and 21 potentially novel transcripts (Table 4).

\section{Discussion}

\section{Protein metabolism}

Anabolic hormones stimulate muscle growth mainly by increasing protein synthesis (Rooyackers \& Nair 1997). In this study, GDX repressed the expression of heat shock protein family member 7 (Hspb7), whereas DHT injection up-regulated five genes encoding ribosomal proteins and chaperones (Mrpl51, Rpl34, Rps20, Cct8 and Cabcl) within $3 \mathrm{~h}$ as well as modulating three other transcripts (Fxr2h, Rps24 and Rps27) at $24 \mathrm{~h}$. In addition to protein synthesis, Rpl34 and Rps20 are implicated in polyamine biosynthesis (Panagiotidis et al. 1995). The Cict8 protein whose expression is highly dependent on cell growth (Yokota et al. 1999) folds newly synthesized proteins including cellular components necessary for cell growth (Thulasiraman et al. 1999) as well as behaving as microtubule-associated protein (Roobol et al. 1999). Mrpl51 is encoded by mitochondrial DNA, and Cabcl encodes a mitochondrial protein essential for the proper conformation and functioning of protein complexes in the respiratory chain (Iiizumi et al. 
Table 1 Components of muscle contraction, extracellular matrix, transcription and protein metabolism regulated by GDX and DHT injection in skeletal muscle

GDX+DHT

Tags ...

\section{Muscle contraction TGGGCCACCTC GTCAGCTGTTG}

Extracellular matrix TCTTCTATGCA

AGTCCACTGGG

CACATAAGACT

Transcription

GGGAAGGTTTT

GTTACAGGTAA

AGTTCACAGAA

Protein metabolism

Ribosomal protein GGAAGTACACA

TGTGCCAAGTG

2

2

\begin{tabular}{|c|c|c|c|c|}
\hline $1 \mathrm{~h}$ & $3 \mathrm{~h}$ & $6 \mathrm{~h}$ & $24 \mathrm{~h}$ & \\
\hline $465 \uparrow$ & $1066 \uparrow$ & 154 & $506 \uparrow$ & Pvalb \\
\hline 4 & 5 & 3 & $15 \uparrow$ & EST colla2 \\
\hline 0 & 0 & 0 & 0 & $\begin{array}{l}\text { Serpina1a, } \\
1 \mathrm{~b} \text { and } 1 \mathrm{e}\end{array}$ \\
\hline
\end{tabular}

$13 \uparrow \quad 0$

0

0

$0 \quad$ Serpina3k

$\begin{array}{lllllll}3 & 3 & 10 & 29 \uparrow & 4 & 9 & \text { Mrp151 }\end{array}$

$\begin{array}{lllllll}9 & 5 & 32 \uparrow & 6 & 12 & \mathrm{Rp} 134\end{array}$

$\begin{array}{lrrrrrrr}\text { CCTACCAAGAC } & 25 \downarrow & 94 & 242 \uparrow & 281 \uparrow & 198 & 159 & \text { Rps20 } \\ \text { GCCTTTATGAG } & 239 & 324 & 256 & 236 & 212 & 134 \downarrow & \text { Rps24 } \\ \text { CACAAACGGTA } & 339 & 426 & 396 & 419 & 457 & 184 \downarrow & \text { Rps27 }\end{array}$

\section{Chaperone}

GCAAAACCAGC

CCCTGCTTCGC$$
1
$$$$
1
$$$$
16 \uparrow
$$$$
9
$$$$
6
$$$$
17 \uparrow \quad \text { Cct8 }
$$$$
33 \uparrow 8
$$$$
9
$$$$
24
$$$$
17
$$$$
25
$$

Hspb7

\section{Degradation}

$\begin{array}{cccccccc}\text { TCCATCAAGAA } & 4 & 2 & 12 & 22 \uparrow & 5 & 11 & \text { Psmc5 } \\ \text { GAGGGCATCCT } & 7 & 4 & 43 \uparrow & 47 \uparrow & 23 \uparrow & 37 \uparrow & \text { Psmc3 }\end{array}$

\section{Description}

(UniGene cluster, GenBank accession no.)

Parvalbumin (Mm.2766, AK013561)

Triadin (Mm.216837, AK009816)

EST procollagen type I $\alpha 2$ (Mm.277792, CB575147)

Serine (or cysteine) proteinase inhibitor clade A member 1a (Mm.259233, NM_009243), 1b (Mm.275860, NM_009244) and 1e (Mm.89843, AK004999)

Serine (or cysteine) proteinase inhibitor clade A member 3K (Mm.291706, BC016407)

Fragile $\mathrm{X}$ mental retardation gene 2 (Mm.41930, BC062971)

O-linked $\mathrm{N}$-acetylglucosamine transferase (Mm.259191, BC057319)

SET and MYND domain containing 2 (Mm.294629, NM_026796

Mitochondrial ribosomal protein L51 Mm.138749, BC021535); similar to ribosomal protein 64 (Mm.331885, AV006065)

Similar to 60 S ribosomal protein L34 (Mm.291750, XM_357642; Mm.300326, XM_356686; Mm.300786, XM_357496; Mm.300749, XM_357418), RIKEN 1100001122 (Mm.140568, XM_357325) Ribosomal protein S20 (Mm.21938, NM_026147, Mm.313599, XM_142259; Mm.291567, XM_357319)

Ribosomal protein S24 (Mm.295727, XM_354797; Mm.293878, XM_140164; Mm.299799, Al151777)

Ribosomal protein S27 (Mm.301283, NM_027015; Mm.270283, BC055693; Mm.311357, AV105261; Mm.325078; AV123585)

Chaperone $\mathrm{ABC} 1$ activity of bcl complex-like (Mm.38330, NM_023341) Chaperone subunit $8(\theta)(\mathrm{Mm} .1789$, BC009007)

Heat shock protein family member 7 (Mm.46181, NM_013868)

Protease 26S subunit ATPase 5 (Mm.272919, NM_008950)

Protease 26S subunit ATPase 3 (Mm.289832, NM_008948)

\footnotetext{
$\uparrow \downarrow$ Significantly up- and down-regulated compared with GDX; $P<0.05$. Mm, mus musculus.
} 
Table 2 Components of proliferation, cell signalling and other functions regulated by GDX and DHT injection in skeletal muscle

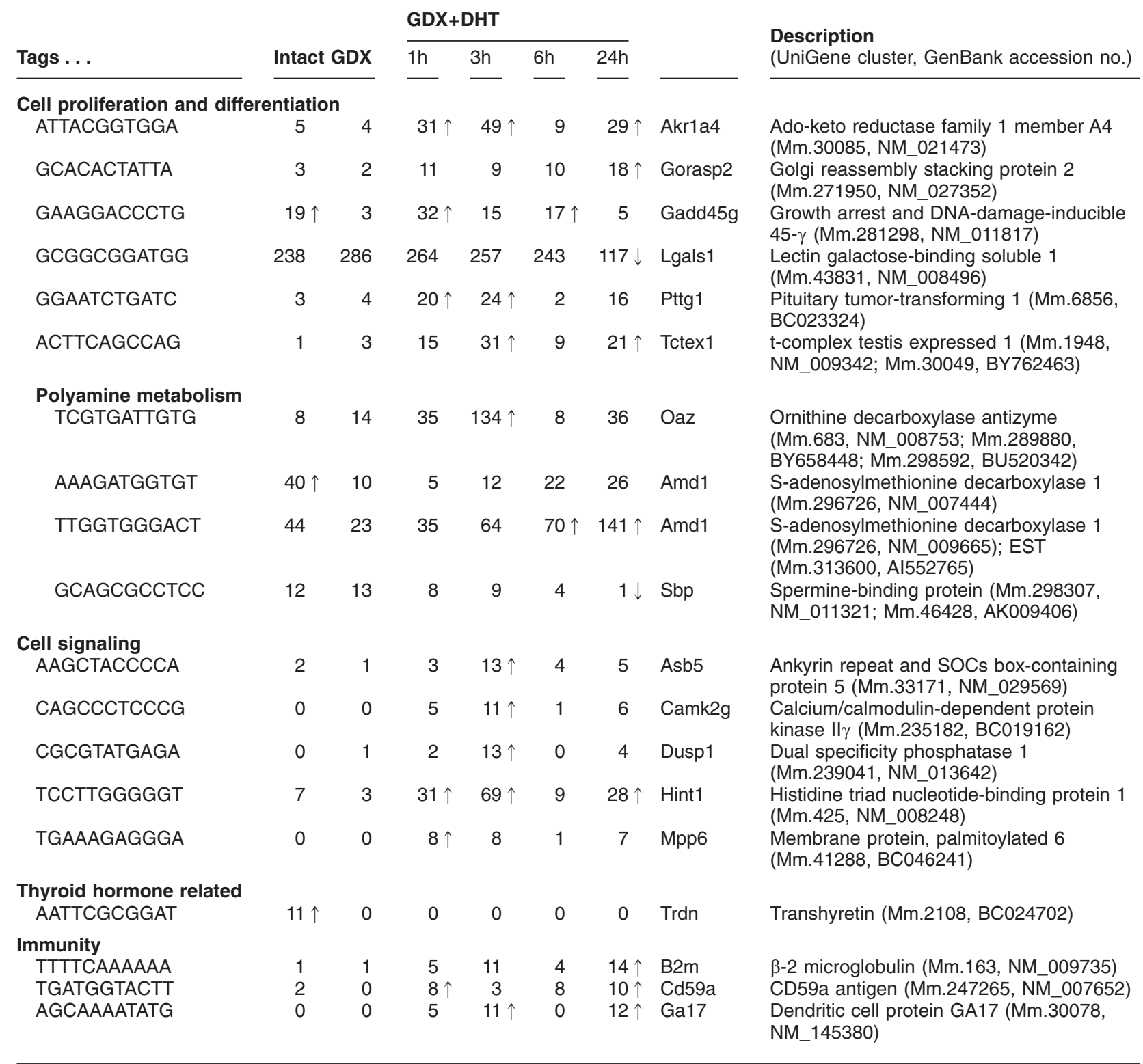

$\uparrow \downarrow$ Significantly up- and down-regulated compared with GDX; $P<0.05$.

2002). Indeed, the expressions of 18 transcripts related to OxPhos and ATP production were up-regulated by DHT in the current study. These data suggest that DHT increases protein synthesis and stabilization in parallel with cell growth within $3 \mathrm{~h}$ in mice in vivo.

The transcripts modulated $24 \mathrm{~h}$ after DHT treatment in the present study suggest the following: (1) the augmentation of mRNA transport between cytoplasm and nucleolus by Fragile X-related proteins (FMRP) (Tamanini et al. 1999), (2) the suppression of mRNA degradation by ribosomal protein S27 (Revenkova et al. 1999) and (3) the decrease in protein synthesis since ribosomal protein S24 is closely involved in both the initiation and elongation processes during protein synthesis (Bommer et al. 1988). The fact that the rate of protein synthesis declines drastically during mitosis in mammalian cells could explain the data.

Degradation of proteins by the $26 \mathrm{~S}$ proteasome is essential for cell cycle progression, polyamine metabolism and class I heavy chain of major histocompatibility complex (MHC) presentation on the cell surface. DHT induced Psmc3 and Psmc5 whose proteins are the 
Table 3 Components of metabolism regulated by GDX and DHT injection in skeletal muscle

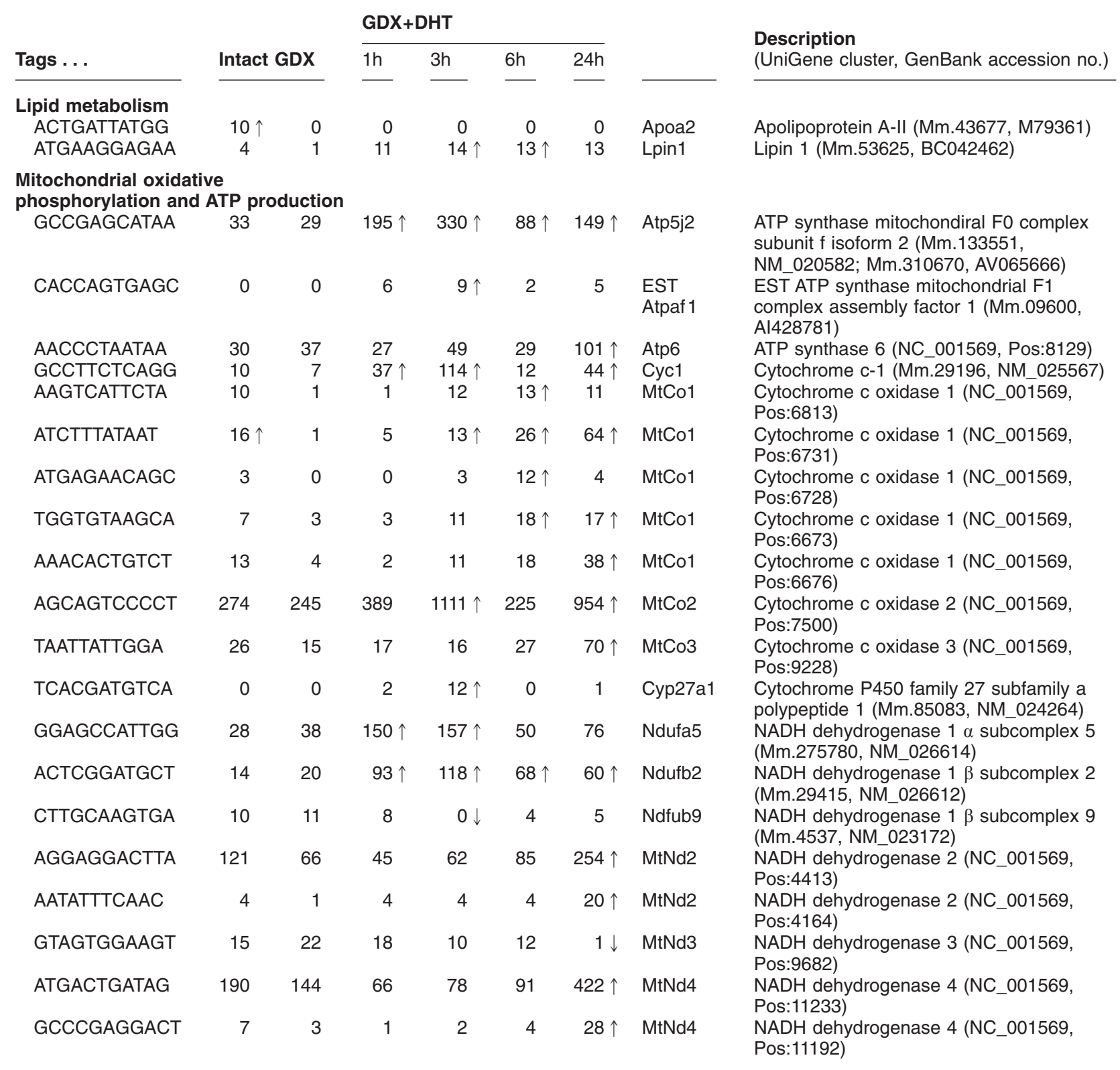

$\uparrow \downarrow$ Significantly up- and down-regulated compared with GDX; $P<0.05$.

integral components of the 19S regulatory subunit of the $26 \mathrm{~S}$ proteasome, which might reflect the induction of cell proliferation and modulation of immunity by DHT as discussed below.

\section{Transcription}

Transcriptional regulation is an essential control point for diverse cellular functions such as cell proliferation, differentiation, transformation and apoptosis. DHT up-regulated five transcriptional factors (Ogt, Pttgl, Psmc3, Psmc5 and Smyd2) within $3 \mathrm{~h}$ after the treatment as well as Fxr2h at $24 \mathrm{~h}$. The activation of the MAPK cascade results in the translocation of cytoplasmic Pttgl protein into the nucleus (Pei 2000) where Pttgl protein transactivates target genes which promote cell proliferation (Pei 2001). The proteins of Psmc3 and Psmc5 suggest roles in the transactivation of thyroid 
Table 4 Partially characterized and potential novel genes regulated by GDX and DHT injection in skeletal muscle

\begin{tabular}{|c|c|c|c|c|c|c|c|c|}
\hline \multirow[b]{2}{*}{ Tags ... } & \multirow{2}{*}{\multicolumn{2}{|c|}{ Intact GDX }} & \multicolumn{4}{|c|}{ GDX+DHT } & & \multirow{2}{*}{$\begin{array}{l}\text { Description } \\
\text { (UniGene cluster, GenBank accession no.) }\end{array}$} \\
\hline & & & $1 \mathrm{~h}$ & $3 \mathrm{~h}$ & $6 \mathrm{~h}$ & $24 h$ & & \\
\hline \multicolumn{9}{|c|}{ Partially characterized } \\
\hline GCGACAGAGGT & 0 & 3 & $26 \uparrow$ & $20 \uparrow$ & 4 & 10 & D12Ertd647e & $\begin{array}{l}\text { DNA segment Chr } 12 \text { ERATO Doi } 647 \\
\left(\mathrm{Mm} .2121, \mathrm{NM} \_026790\right)\end{array}$ \\
\hline CAGCCAGTGTA & 2 & 0 & $9 \uparrow$ & 8 & 1 & $12 \uparrow$ & 0610038P07Rik & $\begin{array}{l}\text { RIKEN 0610038P07 (Mm.232728, } \\
\text { NM_026155) }\end{array}$ \\
\hline CTCAATGAGTG & 10 & 4 & $27 \uparrow$ & 12 & 7 & 5 & 1110030N17Rik & $\begin{array}{l}\text { RIKEN 1110030N17 (Mm.266035, } \\
\text { NM_146118) }\end{array}$ \\
\hline GGCTAAAGTTT & 2 & 1 & 1 & 10 & 1 & $11 \uparrow$ & 2310008C07Rik & $\begin{array}{l}\text { RIKEN 2310008C07 (Mm.261043, } \\
\text { BG797002) }\end{array}$ \\
\hline AGTGGCAAGAG & 2 & 4 & $23 \uparrow$ & $52 \uparrow$ & 3 & 18 & 2310015B20Rik & $\begin{array}{l}\text { RIKEN 2310015B20 (Mm.64672, } \\
\text { AK009351) }\end{array}$ \\
\hline ССТTTCAAACC & 5 & 3 & 5 & $24 \uparrow$ & 7 & 4 & 2310016M24Rik & $\begin{array}{l}\text { RIKEN 2310016M24 (Mm.5265, } \\
\text { NM_183256) }\end{array}$ \\
\hline GCCAGACCTG & 3 & 6 & 6 & $63 \uparrow$ & 2 & 6 & 2610205H19Rik & $\begin{array}{l}\text { RIKEN 2610205H19 (Mm.195626, } \\
\text { BC0118324) }\end{array}$ \\
\hline \multicolumn{9}{|l|}{ No match } \\
\hline AAAAATCATCG & $34 \uparrow$ & 5 & 12 & $44 \uparrow$ & $60 \uparrow$ & $70 \uparrow$ & & \\
\hline AACGGCTAAAC & 17 & 29 & 39 & 65 & 47 & $135 \uparrow$ & & \\
\hline ACCCCAGTTCA & 1 & 2 & $18 \uparrow$ & 13 & 9 & $16 \uparrow$ & & \\
\hline AGCAGTCCCCG & 2 & 0 & 3 & $9 \uparrow$ & 3 & 2 & & \\
\hline AGGAAGCTGAG & 1 & 3 & 4 & $20 \uparrow$ & 1 & 3 & & \\
\hline CCCGTTCTCCT & 1 & 0 & 4 & $11 \uparrow$ & 1 & 5 & & \\
\hline GATCCTGCAGA & 0 & 0 & 2 & 6 & 1 & $9 \uparrow$ & & \\
\hline GCTGCCCTCCC & $13 \uparrow$ & 1 & 0 & $15 \uparrow$ & $18 \uparrow$ & 10 & & \\
\hline GCTGCCCTCCT & 7 & 0 & 0 & $13 \uparrow$ & $14 \uparrow$ & $15 \uparrow$ & & \\
\hline GGGATTTAAAA & 3 & 5 & $26 \uparrow$ & $30 \uparrow$ & 11 & $84 \uparrow$ & & \\
\hline TCATTGGTCGC & 10 & 3 & 2 & 11 & $19 \uparrow$ & $19 \uparrow$ & & \\
\hline TCCCCGTACAC & 13 & 8 & 14 & 30 & 24 & $31 \uparrow$ & & \\
\hline TGTACAAACAG & $30 \uparrow$ & 8 & 6 & 13 & 12 & 24 & & \\
\hline CAAACCCTTAG & 11 & 15 & 6 & 4 & $1 \downarrow$ & 3 & & \\
\hline CCGTTTCTTTA & 4 & 10 & 8 & 7 & 4 & $0 \downarrow$ & & \\
\hline GATGATGCTAA & 9 & 11 & 11 & 10 & 2 & $0 \downarrow$ & & \\
\hline GGTGATGCTAA & 10 & 21 & 19 & 19 & 12 & $2 \downarrow$ & & \\
\hline GTCCCTATTAA & 3 & 10 & 8 & 5 & 1 & $0 \downarrow$ & & \\
\hline GTGACCACGGG & 31 & 28 & 23 & 26 & 18 & $6 \downarrow$ & & \\
\hline GTGATGCTGAG & 13 & 26 & 14 & 14 & 9 & $5 \downarrow$ & & \\
\hline TAGAGACTGCC & $3 \downarrow$ & 26 & 30 & 42 & 30 & 17 & & \\
\hline
\end{tabular}

$\uparrow \downarrow$ Significantly up- and down-regulated compared with GDX; $P<0.05$.

hormone receptor (Ishizuka et al. 2001). GDX also decreased the expression level of Ttr, whose product is a homotetrameric plasma protein which carries thyroxine as well as retinol. Although thyroid hormones are essential during growth, both an excess and a deficiency cause muscle wasting by unknown mechanisms (Rooyackers \& Nair 1997). Fxr2h protein shows a strong transcription activation (Hillman \& Gecz 2001). Nuclear and cytoplasmic protein glycosylation is a widespread and reversible post-translational modification in eukaryotic cells. Intracellular glycosylation of serine and threonine residues is catalyzed by the protein of Ogt, which regulates a number of cellular functions including transcriptional activation (p53 target genes)/repression (RNA polymerase II) and translational activation (Wells et al. 2003). The presence in the Smyd2 gene of SET and
MYND domains would be in agreement with effects on histone deacetylation and methylation respectively (Sims et al. 2002). Thus, our results suggest that at least some of the actions of DHT occur through activation or repression of the transcriptional regulators.

\section{Calcium ion, muscle contraction and cell proliferation}

In the skeletal muscle, $\mathrm{Ca}^{2+}$ plays a key role in contraction and relaxation. The present study showed that DHT treatment increased the expression of Pvalb, a high affinity $\mathrm{Ca}^{2+}$-binding protein acting as a muscle relaxing factor after contraction, and Trdn which forms a quaternary complex with ryanodine receptor, junctin and calsequestrin in the lumen of sarcoplasmic reticulum (SR) for the passive buffering of SR luminal $\mathrm{Ca}^{2+}$ as well 
as an active $\mathrm{Ca}^{2+}$ release from SR process during excitation-contraction coupling. Transgenic mice overexpressing $\operatorname{Trdn} 1$ in the heart show cardiac hypertrophy with impaired relaxation and blunted contractility (Kirchhefer et al. 2001). Thus, induction of both muscle relaxing and contracting factors might contribute to a power generation which is generally observed in athletes who are taking anabolic steroids.

Depolarization of muscle cells also results in a slow transient release of $\mathrm{Ca}^{2+}$, which is mediated by phospholipase $\mathrm{C}$ (PLC) and $\mathrm{IP}_{3}$ via $\mathrm{IP}_{3}$ receptors (Estrada et al. 2001, Powell et al. 2001), and leads to the phosphorylation of ERK1/2 (Powell et al. 2001). In osteoblasts, DHT activates $\mathrm{G}_{4}$ protein coupled to PLC- $\beta$ which increases formation of $\mathrm{IP}_{3}$ and diacylglycerol (DAG), and triggers the releases of intracellular $\mathrm{Ca}^{2+}$ from endoplasmic reticulum (Zagar et al. 2004). The increases in DAG and $\mathrm{Ca}^{2+}$ levels regulate the activity of protein kinase $\mathrm{C}(\mathrm{PKC})$ which stimulates ERK1/2 via the activation of MAPK kinase 1/2 (Zagar et al. 2004). The current results have shown the inductions of Camk2g, Duspl and Hintl within $3 \mathrm{~h}$ after DHT injection. Multifunctional $\mathrm{Ca}^{2+} /$ calmodulindependent protein kinase II (CaMKII) mediates cellular responses to intracellular $\mathrm{Ca}^{2+}$ and is implicated in the control of essential functions such as synaptic transmission, ion channels, gene transcription and cell cycle progression (Santella 1998, Anderson 2005). Proliferating cells (Tombes \& Krystal 1997) and hypertrophied heart with enhanced contractility (Colomer et al. 2003) express CaMKII $\gamma$ isoform which is encoded by Camk2g. Duspl (also named CL100 or MAPK phosphatase 1) was originally identified as an immediate early gene induced by mitogens (Charles et al. 1992, Keyse \& Emslie 1992), and its transcription level reflects the activation of ERK1/2 (Camps et al. 2000). Hint1, a PKC-interacting protein which was originally thought to inhibit PKC, may play a tumor-suppressor role (Su et al. 2003). We have observed the up-regulation of Mpp6, Oaz, Psmc3 and Psmc5 within $3 \mathrm{~h}$ by DHT treatment. The function of Mpp6, a member of the p55-like membrane-associated guanylate kinase (MAGUK) subfamily, is not yet known. However, MAGUK interacts with glutamate receptors and various ionic channels (Godreau et al. 2004). Polyamines (spermine, spermidine and putrescine) also interact with certain ion channels and control intracellular $\mathrm{Ca}^{2+}$ levels (Williams 1997). The polyamine biosynthesis in mammalian cells begins with a production of putrescine by ornithine decarboxylase (ODC). When intracellular polyamine levels increase, ODG antizyme, encoded by Oaz, binds to ODC and facilitates its rapid degradation by the $26 \mathrm{~S}$ proteosome (Thomas \& Thomas 2003). Thus, the inductions of Oaz as well as Psmc3 and Psmc5, which are integral components of the $26 \mathrm{~S}$ proteasome, may reflect the increased level of intracellular polyamines.
Moreover, the modulations of these transcripts as well as Mpp6 within $3 \mathrm{~h}$, the same time-course as Camk2g, suggest their participation in controlling intracellular $\mathrm{Ca}^{2+}$. In addition, the Ras/MAPK pathway controls the transcription of Cict8 (Yamazaki et al. 2003) which was induced at $1 \mathrm{~h}$ after DHT treatment in the present study. Taken together, the second messenger, namely intracellular $\mathrm{Ca}^{2+}$, and its downstream cascades including PKC and MAPK, which are essential for the regulation of cell growth, seem to be modulated by DHT.

Satellite cells/myoblasts within the skeletal muscle tissue proliferate upon exposure to growth factors and following muscle injury, but they stop dividing once they fuse to pre-existing muscle fibers. The fusion is generally coupled with onset of cell proliferation. In the present study, DHT up-regulated transcripts related to S-phase entry (Pttg1) (Nasmyth et al. 2000), microtubule assembly (Cct8) (Roobol et al. 1999), bipolar spindle formation (Tctex1) (Vaisberg et al. 1993), equal chromosomal segregation (Pttgl) (Nasmyth et al. 2000), stacking of Golgi cisternae (Gorasp2) (Shorter et al. 1999) and detoxification of reactive metabolites produced during cell proliferation (Akrla4) (Barski et al. 2004), suggesting an induction of cell proliferation by DHT. On the other hand, DHT down-regulated a cystostatic factor, Lgals1, which maintains $G_{0}$ and controls $G_{2}$ traverse (Wells \& Mallucci 1991). The mitotic spindle requires the assembly/disassembly of microtubules and action of motor complex such as dynein (Vaisberg et al. 1993). Tctexl protein is a light chain of the dynein motor complex (Tai et al. 1998). The Cct8 increases during $\mathrm{G}_{1} / \mathrm{S}$ transition through the early $\mathrm{S}$ phase (Yokota et al. 1999). Pttgl, human securing, accumulates at the onset of the $\mathrm{S}$ phase and peaks at the $\mathrm{G}_{2}-\mathrm{M}$ phases, and prevents premature separin activation during mitosis (Nasmyth et al. 2000).

DHT up-regulated Psmc3, Dusp1, Gadd45g and Pttgl in the present conditions. Overexpression of Psmc3 increases p53 and p21 proteins (Pollice et al. 2004). In response to DNA damage and other stresses, the tumor suppressor p53 induces either cell cycle arrest or apoptosis depending on specific cellular contexts $(\mathrm{Yu}$ \& Zhang 2005). In response to DNA damage, p53 promotes DNA repair by affecting the DNA excision repair pathway and by arresting cells in $G_{1}$ through induction of p21 which contribute to providing more time for the repair (Smith \& Seo 2002), whereas p53-mediated $\mathrm{G}_{1}$ arrest also occurs through induction of Duspl in the absence of DNA damage ( $\mathrm{Li}$ et al. 2003). Protein encoded by Gadd45g interacts with p21 and suppresses cell growth without any evidence of apoptosis (Nakayama et al. 1999). The growth arrest mediated by the cell cycle inhibitors p21 and Gadd45 inhibit the apoptotic response induced by apoptotic targets of p53 (Yu \& Zhang 2005). Moreover, the securing encoded by Pttgl inhibits the ability of p53 to induce cell death 
(Bernal et al. 2002). Taken together, DHT might promote $G_{1}$ arrest without inducing apoptosis in the current study.

The current study reports the induction of Amdl which encodes S-adenosylmethionine decarboxylase (SAMDC), Oaz, Rps20 and Rpl34 as well as Psmc3 and Psmc5 after DHT injection. On the other hand, DHT down-regulated Sbp whose product correlates with spermine accumulation (Moruzzi et al. 1982). Polycationic compounds synthesized by the rate-limiting enzymes, ODC and SAMDC, are crucial for growth and proliferation of mammalian cells. The ODC antizyme encoded by Oaz, as well as ribosomal proteins L34 and S20, inhibit ODC and arginine decarboxylases (Panagiotidis et al. 1995). The ODC and SAMDC are degraded by the 26S proteasome (Yerlikaya \& Stanley 2004) which is encoded by Psmc3 and Psmc5. The ODC antizyme also inhibits polyamine uptake and stimulates excretion (Sakata et al. 2000). Moreover, polyamine uptake is inhibited by PKC and is stimulated by its inhibition (Dot et al. 2000). Coincidentally, Hintl, which inhibits PKC, had a similar pattern of modulation to that of Oaz with DHT treatment. Taken together, the modulations of Pttgl, Cct8, Tctex1, Gorasp2, Akrla4, Lgals1, Amd1, Oaz, Rpl34, Rps20, Psmc3, Psmc5 and Sbp by DHT treatment in the present study might reflect proliferation of satellite cells/myoblasts in the skeletal muscle.

In addition, Pttgl protein induces angiogenesis both in vitro and in vivo (Ishikawa et al. 2001). In the present study, DHT up-regulated Pttgl and Asb5 which is a novel protein implicated in the initiation of arteriogenesis (Boengler et al. 2003). Vascularization is an important determinant of energy supply and waste removal during muscle contraction, and its stimulation by DHT in this study is thus in agreement with other data presented above.

\section{Metabolism}

In lipid metabolism, GDX shut down Apoa2 and DHT up-regulated Lpin1. Apolipoprotein A-II encoded by Apoa2, the second most abundant protein of high density lipoprotein (HDL) particles, exerts a marked effect on HDL binding and selective lipid uptake by class B scavenger receptors. In the mouse, enhanced Lpin 1 expression in the skeletal muscle promotes obesity by decreasing whole body energy expenditure and fat utilization as well as by inducing insulin resistance (Phan \& Reue 2005). In contrast to what happens in the muscle, overexpression of Lpin 1 in the adipose tissue causes obesity without insulin resistance (Phan \& Reue 2005). We have previously reported that the expression level of Lpin 1 in adipose tissue remains unaltered with DHT treatment (Bolduc et al. 2004). The induction of Lpin 1 only in the muscle might suggest that carbohydrate was used for the increased OxPhos and ATP production. Further studies will be needed to clarify this intriguing mechanism.

\section{Immunity}

DHT increased B2m, Cd59a and Ga17 in the present study. The human homologue of Gal7 (also named hfl-B5) encodes a cell surface membrane fusion protein which enables entry and infection of herpes simplex virus (Perez et al. 2005). $\beta_{2} \mathrm{M}$ encoded by the $\mathrm{B} 2 \mathrm{~m}$ gene is a small immunoglobulin-like protein which is non-covalently associated with the class I heavy chain of MHC molecules. Human skeletal muscle fibers do not show cytochemically demonstrable expression of class I MHC or $\beta_{2} \mathrm{M}$ while these proteins are regularly present in regenerating muscle after chronic partial denervation, Duchenne dystrophy and several types of inflammatory myopathy (Karpati et al. 1988). Moreover, $\beta_{2} \mathrm{M}$ is a bone-derived growth factor which stimulates bone DNA and collagen synthesis (Canalis et al. 1987). Although the effects of $\beta_{2} \mathrm{M}$ in the skeletal muscle are not known, the present study has reported the up-regulation of B2m and EST Colla2 $24 \mathrm{~h}$ after DHT injection. Coincidentally, Psmc3 which suppresses HIV Tatmediated transactivation (Nelbock et al. 1990) was also up-regulated, whereas HIV Tat is known to repress the transcription of the $\beta_{2} \mathrm{M}$ promoter (Carroll et al. 1998). Thus, the up-regulation of Psmc3 by DHT might have induced B2m and EST Colla2 in the current conditions. The myogenic process is heavily dependent upon cell migrations and adhesions. Collagen, an adhesive ECM component, binds to substrates like fibronectin, promoting cell-substrate adhesion which is a requisite for cell migration. During proliferation, satellite cells adhere to their substrate by expressing high levels of collagen type I and are beginning the process of cell migration, which leads to myoblast alignment and subsequent fusion (Velleman \& McFarland 1999). Thus, the modulations of $\mathrm{B} 2 \mathrm{~m}$ and EST Colla2 by DHT treatment might suggest that myoblast proliferation (migration and adhesion) occurs $24 \mathrm{~h}$ after DHT stimulation in mice in vivo. In order to prevent a complement-mediated attack, host tissue expresses a variety of regulatory proteins that limit damage to the pathogen cell surface. The complement regulatory protein CD59 is a glycosylphosphatidylinositol-anchored membrane protein which inhibits the formation of membrane attack complex (MAC) and protects the cells from MAC-induced lysis (Turnberg \& Botto 2003). In comparison with control muscle, atrophied muscle fibers of inflammatory myopathic patients do not contain CD59 whereas regenerating muscle cells (myoblasts and myotubes) from the same patients have a strong immunostaining with CD59 antibody (Gendek-Kubiak \& Gendek 2004). From the facts that (1) $\beta_{2} \mathrm{M}$ and CD59 are commonly highly expressed in myoblasts and regenerating fibers, 


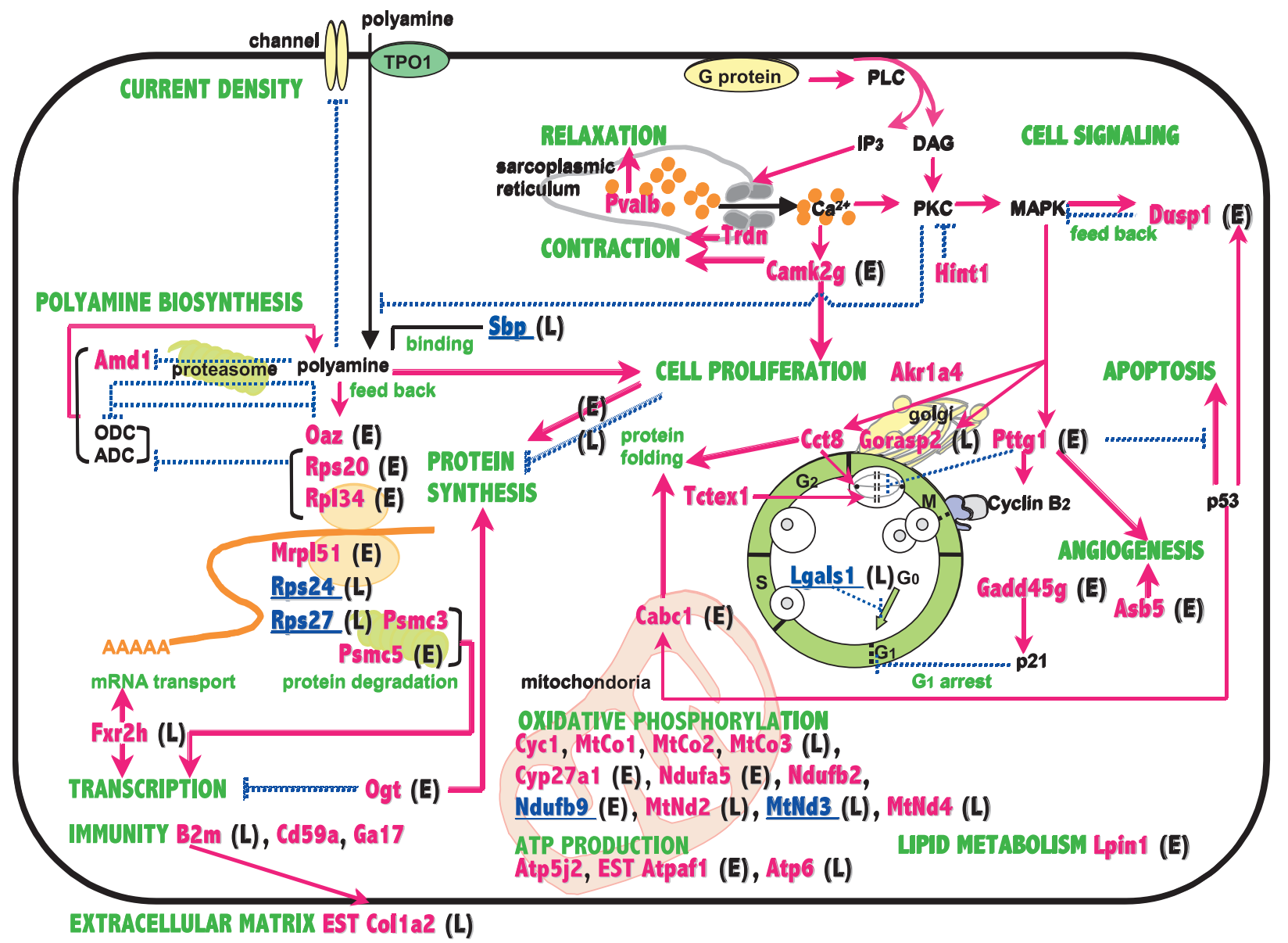

Figure 1 Overview of ARGs in the skeletal muscle. The up- and down-regulated transcripts by DHT treatment are shown in red and blue (underlined) characters respectively. Transcripts showing only early (DHT $1 \mathrm{~h}, 3 \mathrm{~h}$ and $6 \mathrm{~h}$ ) or late (DHT $24 \mathrm{~h}$ ) response are noted as (E) or (L) respectively. The solid and dotted lines represent activation/induction and inhibition, respectively. Akr1a4, aldo-keto reductase family 1 member A4; Amd1, S-adenosylmethionine decarboxylase 1; Asb5, ankyrin repeat and SOCs box-containing protein 5; Atp5j2, ATP synthase mitochondrial F0 complex subunit $\mathrm{f}$ isoform 2; Atp6, ATP synthase 6; Atpaf1, ATP synthase mitochondrial F1 complex assembly factor 1 ; B2m, $\beta-2$ microglobulin; Cabc1, chaperone ABC1 activity of bc1 complex like; Camk2g, calcium/calmodulin-dependent protein kinase Il $\gamma$; Cct8, chaperonin subunit 8; Cd59a, CD59a antigen; Col1a2, procollagen type I $\alpha 2$; Cyc1, cytochrome c-1; Cyp27a1, cytochrome P450 family 27 subfamily a polypeptide 1; DAG, diacylglycerol; Dusp1, dual specificity phosphatase 1; EST, expressed sequence tags; Fxr2h, fragile X mental retardation gene 2; Ga17, dendritic cell protein GA17; Gadd45g, growth arrest and DNA damage-inducible 45 $;$; Gorasp2, Golgi reassembly stacking protein 2; Hint1, histidine triad nucleotide-binding protein 1; IP , inositol 1,4,5-trisphosphate; Lgals1, lectin galactose-binding soluble 1; Lpin1, lipin 1; MAPK, mitogen-activated protein kinase; Mrpl51, ribosomal protein L51; MtCo1, cytochrome c oxidase 1; MtCo2, cytochrome c oxidase 2; MtCo3, cytochrome c oxidase 3; MtNd2, NADH dehydrogenase 2; MtNd3, NADH dehydrogenase 3; MtNd4, NADH dehydrogenase 4; Ndufa5, NADH dehydrogenase $1 \alpha$ subcomplex 5; Ndufb2, NADH dehydrogenase $1 \beta$ subcomplex 2; Ndufb9, NADH dehydrogenase $1 \beta$ subcomplex 9; Oaz, ornithine decarboxylase antizyme; Ogt, O-linked N-acetylglucosamine transferase; PKC, protein kinase C; PLC, phospholipase C; Psmc, proteasome 26S subunit ATPase; Pvalb, parvalbumin; Rp, ribosomal protein; Pttg1, pituitary tumor-transforming 1; Sbp, spermine-binding protein; Tctex1, t-complex testis expressed 1; TPO1, polyamine transporter; Trdn, triadin.

(2) muscle regeneration induced by muscle injury and hypertrophy induced by growth stimuli share common processes of muscle growth and (3) many transcripts modulated at $24 \mathrm{~h}$ after DHT treatment in the current study were related to cell proliferation (mitosis), the elevated expression levels of B2 m and Cd59a $24 \mathrm{~h}$ after DHT injection might reflect myoblast proliferation.

\section{Conclusion}

An overview of ARG in skeletal muscle is presented in Fig. 1. The early responses to DHT injection (DHT 1, 3 and $6 \mathrm{~h}$ ) are the induction of both muscle relaxing (Pvalb) and contracting (Trdn) factors which modulate intracellular $\mathrm{Ca}^{2+}$ levels. DHT also induced the 
transcripts related to cell signaling such as $\mathrm{Ca}^{2+}$ (Camk2g), PKC (Hintl) and MAPK pathways (Duspl) as well as polyamine biosynthesis (Amd1, Oaz, Psmc3, Rps20 and Rpl34), cell proliferation (Akrla4, Cct8, Pttg1 and Tctexl), cell cycle arrest (Gadd45g), p53 (Cabc1, Duspl and Pttg1) and angiogenesis (Asb5 and Pttg1). The induction of mRNAs related to transcription (Ogt, Psmc3 and Psmc5), protein synthesis (Mrpl51, Ogt, Rps20 and Rpl34), modification (Cabc1, Get8 and Ogt) and degradation (Psmc3 and Psmc5), oxidative phosphorylation (Cyc1, MtCol, MtCo2, Cyp27al, Ndufa5 and Ndufb2), ATP production (Atp5j2 and EST Atpafl), and lipid metabolism (Lpin1) as well as immunity (Cd59a and Ga17) have also been observed at these time-points. However, the induction of transcripts related to $\mathrm{Ca}^{2+}$ signaling, MAPK, cell cycle arrest, p53, protein synthesis and angiogenesis are not significant any more $24 \mathrm{~h}$ after DHT injection while transcripts related to cell cycle progression are still up-regulated. Moreover, the transcripts related to protein synthesis (Rps24 and Rps27) were down-regulated. These results indicate that DHT injection induces power generation, protein synthesis, mitochondrial function and satellite cell/ myoblast proliferation at the transcriptional level in vivo, supporting previous findings of an anabolic action of androgen.

\section{Acknowledgements}

We would like to thank Jean-François Cadrin-Girard, Lin Gan and all the research assistants involved in the ATLAS project for their skillful technical assistance. M Y is supported by the Heart and Stroke Foundation of Canada, the Canadian Institute of Health Research and the Canadian Diabetes Association as a postdoctoral fellow. J S-A is an investigator supported by the Fonds de la recherche en santé du Québec. This work was supported by Genome Québec and Genome Canada. The authors declare that there is no conflict of interest that would prejudice the impartiality of this scientific work.

\section{References}

Adams MD, Kerlavage AR, Fleischmann RD, Fuldner RA, Bult CJ, Lee NH, Kirkness EF, Weinstock KG, Gocayne JD, White O et al. 1995 Initial assessment of human gene diversity and expression patterns based upon 83 million nucleotides of cDNA sequence. Nature 377 3-174.

Anderson ME 2005 Calmodulin kinase signaling in heart: an intriguing candidate target for therapy of myocardial dysfunction and arrhythmias. Pharmacological Therapy 106 39-55.

Araya R, Liberona JL, Cardenas JC, Riveros N, Estrada M, Powell JA, Carrasco MA \& Jaimovich E 2003 Dihydropyridine receptors as voltage sensors for a depolarization-evoked, $\mathrm{IP}_{3} \mathrm{R}$-mediated, slow calcium signal in skeletal muscle cells. Fournal of Genetic Physiology 121 3-16.

Barski OA, Papusha VZ, Kunkel GR \& Gabbay KH 2004 Regulation of aldehyde reductase expression by STAF and CHOP. Genomics 83 119-129.

Bernal JA, Luna R, Espina A, Lazaro I, Ramos-Morales F, Romero F, Arias C, Silva A, Tortolero M \& Pintor-Toro JA 2002 Human securin interacts with p53 and modulates p53-mediated transcriptional activity and apoptosis. Nature Genetics 32 306-311.

Bhasin S, Woodhouse L \& Storer TW 2001 Proof of the effect of testosterone on skeletal muscle. Fournal of Endocrinology 170 27-38.

Boengler K, Pipp F, Fernandez B, Richter A, Schaper W \& Deindl E 2003 The ankyrin repeat containing SOCS box protein 5: a novel protein associated with arteriogenesis. Biochemical and Biophysical Research Communication 302 17-22.

Bolduc C, Larose M, Yoshioka M, Ye P, Belleau P, Labrie C, Morissette J, Raymond V, Labrie F \& St-Amand J 2004 Effects of dihydrotestosterone on adipose tissue measured by serial analysis of gene expression. Fournal of Molecular Endocrinology 33 429-444.

Bommer UA, Stahl J, Henske A, Lutsch G \& Bielka H 1988 Identification of proteins of the $40 \mathrm{~S}$ ribosomal subunit involved in interaction with initiation factor eIF-2 in the quaternary initiation complex by means of monospecific antibodies. FEBS Letters 233 114-118.

Camps M, Nichols A \& Arkinstall S 2000 Dual specificity phosphatases: a gene family for control of MAP kinase function. FASEB Fournal 14 6-16.

Canalis E, McCarthy T \& Centrella M 1987 A bone-derived growth factor isolated from rat calvariae is beta 2 microglobulin. Endocrinology 121 1198-1200.

Carroll IR, Wang J, Howcroft TK \& Singer DS 1998 HIV Tat represses transcription of the beta 2-microglobulin promoter. Molecular Immunology 35 1171-1178.

Charles CH, Abler AS \& Lau LF 1992 cDNA sequence of a growth factor-inducible immediate early gene and characterization of its encoded protein. Oncogene 7 187-190.

Colomer JM, Mao L, Rockman HA \& Means AR 2003 Pressure overload selectively up-regulates Ca2+/calmodulin-dependent protein kinase II in vivo. Molecular Endocrinology 17 183-192.

Dinel S, Bolduc C, Belleau P, Boivin A, Yoshioka M, Calvo E, Piedboeuf B, Snyder EE, Labrie F \& St-Amand J 2005

Reproducibility, bioinformatic analysis and power of the SAGE method to evaluate changes in transcriptome. Nucleic Acids Research 33 e26 1-8.

Dot J, Lluch M, Blanco I \& Rodriguez-Alvarez J 2000 Polyamine uptake in cultured astrocytes: characterization and modulation by protein kinases. Fournal of Neurochemistry 75 1917-1926.

Estrada M, Liberona JL, Miranda M \& Jaimovich E 2000 Aldosterone- and testosterone-mediated intracellular calcium response in skeletal muscle cell cultures. American Fournal of Physiology - Endocrinology and Metabolism 279 E132-E139.

Estrada M, Cardenas C, Liberona JL, Carrasco MA, Mignery GA, Allen PD \& Jaimovich E 2001 Calcium transients in 1B5 myotubes lacking ryanodine receptors are related to inositol trisphosphate receptors. Fournal of Biological Chemistry 276 22868-22874.

Estrada M, Espinosa A, Muller M \& Jaimovich E 2003 Testosterone stimulates intracellular calcium release and mitogen-activated protein kinases via a $G$ protein-coupled receptor in skeletal muscle cells. Endocrinology 144 3586-3597.

Gendek-Kubiak H \& Gendek EG 2004 Immunolocalization of protectin (CD59) and macrophages in polymyositis and dermatomyositis. Fournal of Neuroimmunology 149 187-194.

Godreau D, Neyroud N, Vranckx R \& Hatem S 2004 MAGUKs: not only anchoring proteins. Medical Science $2084-88$.

Herbst KL \& Bhasin S 2004 Testosterone action on skeletal muscle. Current Opinion in Clinical Nutrition and Metabolic Care 7 271-277. 
Hillman MA \& Gecz J 2001 Fragile XE-associated familial mental retardation protein 2 (FMR2) acts as a potent transcription activator. Fournal of Human Genetics 46 251-259.

Iiizumi M, Arakawa H, Mori T, Ando A \& Nakamura Y 2002 Isolation of a novel gene, CABC1, encoding a mitochondrial protein that is highly homologous to Yyast activity of bcl complex. Cancer Research 62 1246-1250.

Ishikawa H, Heaney AP, Yu R, Horwitz GA \& Melmed S 2001 Human pituitary tumor-transforming gene induces angiogenesis. Journl of Clinical Endocrinology and Metabolism 86 867-874.

Ishizuka T, Satoh T, Monden T, Shibusawa N, Hashida T, Yamada M \& Mori M 2001 Human immunodeficiency virus type 1 Tat binding protein-1 is a transcriptional coactivator specific for TR. Molecular Endocrinology 15 1329-1343.

Karpati G, Pouliot Y \& Carpenter S 1988 Expression of immunoreactive major histocompatibility complex products in human skeletal muscles. Annals of Neurology 23 64-72.

Keyse SM \& Emslie EA 1992 Oxidative stress and heat shock induce a human gene encoding a protein-tyrosine phosphatase. Nature $359644-647$.

Kirchhefer U, Neumann J, Baba HA, Begrow F, Kobayashi YM, Reinke U, Schmitz W \& Jones LR 2001 Cardiac hypertrophy and impaired relaxation in transgenic mice overexpressing triadin 1. Journal of Biological Chemistry 276 4142-4149.

Lash AE, Tolstoshev CM, Wagner L, Schuler GD, Strausberg RL, Riggins GJ \& Altschul SF 2000 SAGEmap: a public gene expression resource. Genome Research 10 1051-1060.

Li M, Zhou JY, Ge Y, Matherly LH \& Wu GS 2003 The phosphatase MKP1 is a transcriptional target of p53 involved in cell cycle regulation. Fournal of Biological Chemistry 278 41059-41068.

Michel G \& Baulieu EE 1980 Androgen receptor in rat skeletal muscle: characterization and physiological variations. Endocrinology 107 2088-2098.

Moruzzi MS, Monti MG, Piccinini G \& Mezzetti G 1982 Spermine-binding protein and polyamine metabolism in duodenal mucosa of chick embryo. Life Science 31 1639-1644.

Nakayama K, Hara T, Hibi M, Hirano T \& Miyajima A 1999 A novel oncostatin M-inducible gene OIG37 forms a gene family with MyD118 and GADD45 and negatively regulates cell growth. Fournal of Biological Chemistry $27424766-24772$.

Nasmyth K, Peters JM \& Uhlmann F 2000 Splitting the chromosome: cutting the ties that bind sister chromatids. Science 288 1379-1385.

Nelbock P, Dillon PJ, Perkins A \& Rosen CA 1990 A cDNA for a protein that interacts with the human immunodeficiency virus Tat transactivator. Science 248 1650-1653.

Panagiotidis CA, Huang SC \& Canellakis ES 1995 Relationship of the expression of the S20 and L34 ribosomal proteins to polyamine biosynthesis in Escherichia coli. International fournal of Biochemistry and Cell Biology 27 157-168.

Pei L 2000 Activation of mitogen-activated protein kinase cascade regulates pituitary tumor-transforming gene transactivation function. Fournal of Biological Chemistry 275 31191-31198.

Pei L 2001 Identification of c-myc as a down-stream target for pituitary tumor-transforming gene. Fournal of Biological Chemistry $2768484-8491$.

Perez A, Li QX, Perez-Romero P, Delassus G, Lopez SR, Sutter S, McLaren N \& Fuller AO 2005 A new class of receptor for herpes simplex virus has heptad repeat motifs that are common to membrane fusion proteins. Fournal of Virology 79 7419-7430.

Phan J \& Reue K 2005 Lipin, a lipodystrophy and obesity gene. Cell Metabolism 1 73-83.

Pollice A, Nasti V, Ronca R, Vivo M, Lo Iacono M, Calogero R, Calabro V \& La Mantia G 2004 Functional and physical interaction of the human ARF tumor suppressor with Tat-binding protein-1. Fournal of Biological Chemistry 279 6345-6353.
Powell JA, Carrasco MA, Adams DS, Drouet B, Rios J, Muller M, Estrada M \& Jaimovich E $2001 \mathrm{IP}_{3}$ receptor function and localization in myotubes: an unexplored $\mathrm{Ca}^{2+}$ signaling pathway in skeletal muscle. Foumal of Cell Science 114 3673-3683.

Revenkova E, Masson J, Koncz C, Afsar K, Jakovleva L \& Paszkowski J 1999 Involvement of Arabidopsis thaliana ribosomal protein S27 in mRNA degradation triggered by genotoxic stress. EMBO fournal 18 490-499.

Roobol A, Sahyoun ZP \& Carden MJ 1999 Selected subunits of the cytosolic chaperonin associate with microtubules assembled in vitro. fournal of Biological Chemistry 274 2408-2415.

Rooyackers OE \& Nair KS 1997 Hormonal regulation of human muscle protein metabolism. Annual Reviews in Nutrition 17 457-485.

St-Amand J, Okamura K, Matsumoto K, Shimizu S \& Sogawa Y 2001 Characterization of skeletal muscle in control and immobilized legs. An overview from genetic engineering. FASEB Fournal $15684-692$.

Sakata K, Kashiwagi K \& Igarashi K 2000 Properties of a polyamine transporter regulated by antizyme. Biochemical fournal 347 297-303.

Santella L 1998 The role of calcium in the cell cycle: facts and hypotheses. Biochemical and Biophysical Research Communications 244 317-324.

Shorter J, Watson R, Giannakou ME, Clarke M, Warren G \& Barr FA 1999 GRASP55, a second mammalian GRASP protein involved in the stacking of Golgi cisternae in a cell-free system. EMBO Fournal 18 4949-4960.

Simental JA, Sar M, Lane MV, French FS \& Wilson EM 1991 Transcriptional activation and nuclear targeting signals of the human androgen receptor. Fournal of Biological Chemistry 266 510-518.

Sims RJ 3rd, Weihe EK, Zhu L, O'Malley S, Harriss JV \& Gottlieb PD $2002 \mathrm{~m}$-Bop, a repressor protein essential for cardiogenesis, interacts with skNAC, a heart- and muscle-specific transcription factor. Fournal of Biological Chemistry $27726524-26529$.

Smith ML \& Seo YR 2002 p53 regulation of DNA excision repair pathways. Mutagenesis 17 149-156.

Su T, Suzui M, Wang L, Lin CS, Xing WQ \& Weinstein IB 2003 Deletion of histidine triad nucleotide-binding protein $1 /$ PKC-interacting protein in mice enhances cell growth and carcinogenesis. PNAS $1007824-7829$.

Tai AW, Chuang JZ \& Sung CH 1998 Localization of Tctex-1, a cytoplasmic dynein light chain, to the Golgi apparatus and evidence for dynein complex heterogeneity. Fournal of Biological Chemistry 273 19639-19649.

Tamanini F, Bontekoe C, Bakker CE, van Unen L, Anar B, Willemsen R, Yoshida M, Galjaard H, Oostra BA \& Hoogeveen AT 1999 Different targets for the fragile X-related proteins revealed by their distinct nuclear localizations. Human Molecular Genetics 8 863-869.

Thomas T \& Thomas TJ 2003 Polyamine metabolism and cancer. Fournal of Cellular and Molecular Medicine 7 113-126.

Thulasiraman V, Yang CF \& Frydman J 1999 In vivo newly translated polypeptides are sequestered in a protected folding environment. EMBO foumal 18 85-95.

Tombes RM \& Krystal GW 1997 Identification of novel human tumor cell-specific CaMK-II variants. Biochimica et Biophysica Acta 1355 281-292.

Truhan AP \& Ahmed AR 1989 Corticosteroids: a review with emphasis on complications of prolonged systemic therapy. Annals of Allergy 62 375-391.

Turnberg D \& Botto M 2003 The regulation of the complement system: insights from genetically-engineered mice. Molecular Immunology 40 145-153.

Vaisberg EA, Koonce MP \& McIntosh JR 1993 Cytoplasmic dynein plays a role in mammalian mitotic spindle formation. Fournal of Cell Biology 123 849-858.

Velculescu VE, Zhang L, Vogelstein B \& Kinzler KW 1995 Serial analysis of gene expression. Science $270484-487$. 
Velleman SG \& McFarland DC 1999 Myotube morphology, and expression and distribution of collagen type I during normal and low score normal avian satellite cell myogenesis. Development and Growth Differentiation 41 153-161.

Wells L, Whelan SA \& Hart GW 2003 O-GlcNAc: a regulatory post-translational modification. Biochemical and Biophysical Research Communications 302 435-441.

Wells V \& Mallucci L 1991 Identification of an autocrine negative growth factor: mouse beta-galactoside-binding protein is a cytostatic factor and cell growth regulator. Cell 64 91-97.

Williams K 1997 Interactions of polyamines with ion channels. Biochemical foumal 325 289-297.

Yamazaki Y, Kubota H, Nozaki M \& Nagata K 2003

Transcriptional regulation of the cytosolic chaperonin theta subunit gene, Cctq, by Ets domain transcription factors Elk-1, Sap-la, and Net in the absence of serum response factor. Fournal of Biological Chemistry 278 30642-30651.

Yerlikaya A \& Stanley BA 2004 S-adenosylmethionine decarboxylase degradation by the $26 \mathrm{~S}$ proteasome is accelerated by substrate-mediated transamination. Fournal of Biological Chemistry 279 12469-12478.

Yokota S, Yanagi H, Yura T \& Kubota H 1999 Cytosolic chaperonin is up-regulated during cell growth. Preferential expression and binding to tubulin at $\mathrm{G}(1) / \mathrm{S}$ transition through early S phase. Fournal of Biological Chemistry 274 37070-37078.

Yoshioka M, Tanaka H, Shono N, Snyder EE, Shindo M \& St-Amand J 2003 Serial analysis of gene expression in the skeletal muscle of endurance athletes compared to sedentary men. FASEB fournal 17 1812-1819.

Yu J \& Zhang L 2005 The transcriptional targets of p53 in apoptosis control. Biochemical and Biophysical Research Communications 331 851-858.

Zagar Y, Chaumaz G \& Lieberherr M 2004 Signaling cross-talk from Gbeta4 subunit to Elk-1 in the rapid action of androgens. Journal of Biological Chemistry 279 2403-2413.

Received 3 October 2005

Accepted 6 December 2005 